J. Dairy Sci. 99:6071-6079

http://dx.doi.org/10.3168/jds.2016-10947

(C) American Dairy Science Association ${ }^{\circledR}, 2016$.

\title{
Rapid quantification of casein in skim milk using Fourier transform infrared spectroscopy, enzymatic perturbation, and multiway partial least squares regression: Monitoring chymosin at work
}

\author{
A. Baum, ${ }^{* 1}$ P. W. Hansen,† L. Nørgaard,† John Sørensen, $\ddagger$ and J. D. Mikkelsen* \\ ${ }^{*}$ Center for BioProcess Engineering, Department of Chemical and Biochemical Engineering, Technical University of Denmark, \\ 2800 Kgs. Lyngby, Denmark \\ †Foss Analytical, Foss Allé 1, DK-3400 Hillerød, Denmark \\ †Arla Foods amba, Innovation, Rørdrumvej 2, 8220 Brabrand, Denmark
}

\section{ABSTRACT}

In this study, we introduce enzymatic perturbation combined with Fourier transform infrared (FTIR) spectroscopy as a concept for quantifying casein in subcritical heated skim milk using chemometric multiway analysis. Chymosin is a protease that cleaves specifically caseins. As a result of hydrolysis, all casein proteins clot to form a creamy precipitate, and whey proteins remain in the supernatant. We monitored the cheese-clotting reaction in real time using FTIR and analyzed the resulting evolution profiles to establish calibration models using parallel factor analysis and multiway partial least squares regression. Because we observed casein-specific kinetic changes, the retrieved models were independent of the chemical background matrix and were therefore robust against possible covariance effects. We tested the robustness of the models by spiking the milk solutions with whey, calcium, and cream. This method can be used at different stages in the dairy production chain to ensure the quality of the delivered milk. In particular, the cheese-making industry can benefit from such methods to optimize production control.

Key words: multiway, parallel factor analysis, cheese, infrared spectroscopy

\section{INTRODUCTION}

Fourier transform infrared (FTIR) spectroscopy and multivariate data analysis represent a versatile partnership for rapid but affordable quantitative analysis of the constituents of (bio-)chemical systems. Nevertheless, quantitative chemometric calibrations may generate misleading results and must be handled with great

\footnotetext{
Received January 26, 2016.

Accepted April 21, 2016.

${ }^{1}$ Corresponding author: aba@kt.dtu.dk
}

care. Occasionally, calibration models are established for constituents that cannot be clearly distinguished from others in the sample matrix. Such models may not be robust and may appear valid only because of collinearities between the analyte and the interfering constituent(s). When measuring casein in milk, for example, the usual amount of casein is approximately $80 \%$ of the total protein content. Casein content can therefore be determined by FTIR measurement of total protein (Hewavitharana and van Brakel, 1997; Etzion et al., 2004). However, these calibrations break down when the casein:total protein ratio varies significantly (Venyaminov and Kalnin, 1990). Instead of measuring the analyte of interest, the test determines the degree of collinearity (or covariance) between the 2 constituents, and accuracy depends on that underlying relationship. Other studies have shown that prediction of individual fatty acids in pork backfat using Raman spectroscopy and partial least squares regressions led to misleading results because iodine was measured instead of fatty acids (Berhe et al., 2016). The established correlations were indirect and governed by underlying covariance effects. Similar problems were reported when quantifying individual fatty acids in bovine milk using FTIR and chemometric methods such as partial least squares regression (Eskildsen et al., 2014).

It is therefore relevant to develop ways of resolving the analyte of interest from the underlying matrix before performing chemometric analysis. Such methods may include any kind of chemical change to the system that enables the analyte to stand out from the remaining constituents, including the sample matrix. Such methods should also be tested for robustness against constituents that have previously shown collinear behavior toward the analyte.

One method was reported recently in which an enzymatic perturbation was introduced to resolve interfering analytes using FTIR spectroscopy by adding a substrate-specific kinetic dimension to the analysis. The 
resulting data indicated yet another (kinetic) mode and were analyzed using numerical tensor decomposition methods such as parallel factor analysis (PARAFAC), Tucker3, or multiway partial least squares (NPLS) regression (Baum et al., 2016). Another method employed excitation emission spectroscopy, using an enzymatic-induced change to discriminate between the collinear-behaving analytes tyrosine and levodopa in human plasma (Xie et al., 2015). The PARAFAC method was used to establish third-order calibration models, yielding extraordinary distinguishing power.

In this paper, we introduce enzymatic perturbation as a tool for resolving interfering collinearities when measuring casein in skim milk using FTIR. Casein is a milk protein that is important for cheese-making. Because cheese yield depends mainly on casein concentration (Wedholm et al., 2006), it is important to develop robust determination methods as a way of optimizing the cheese-making process. Because the spectral fingerprints of casein and whey are hardly distinguishable (Susi and Byler, 1986; Kong and Yu, 2007), chymosin can be used to target the analysis by means of specific enzymatic hydrolysis. Chymosin is a protease found in rennet that catalyzes the conversion of $\kappa$-casein in milk to para-k-casein by removing a glycopeptide from the soluble casein (Lucey and Fox, 1993; Langholm Jensen et al., 2013). As a result of this enzymatic conversion, all caseins coagulate to form a creamy precipitate combined with calcium, and whey proteins remain in the supernatant. The resulting cheese curd coagulation can be measured in situ using FTIR. To visualize the spectral changes, the initial spectrum (corresponding to the entire chemical background) can be subtracted from the entire time-resolved series to yield a spectral landscape that represents the enzyme-induced perturbation. We have used such visualizations in this paper and refer to them as evolution profiles (Baum et al., 2013b). Evolution profiles for 2 proteases are given in Figure 1. Both enzymes - chymosin and metalloprotease Prt1 from Pectobacterium carotovorum - hydrolyze proteins in milk, but they can be distinguished by the fact that chymosin cleaves solely caseins, whereas the metalloprotease is not protein-specific (Feng et al., 2014). The 2 profiles are clearly distinguishable, because parts of the spectral evolution are missing for chymosin, indicating its selectivity toward casein. In addition, the intensity of the spectral evolution stands in relationship with corresponding reaction rates and is, therefore, linked to underlying enzyme kinetics.

We hypothesized that targeted enzymatic perturbation, combined with in situ FTIR measurement and multiway analysis, would represent a powerful methodology for resolving spectral collinearities when estab- lishing quantitative calibration models. Specific casein calibrations would rely on time-resolved measurement of milk coagulation induced by chymosin and would show robustness against varying whey, cream, and calcium levels.

\section{MATERIALS AND METHODS}

\section{Materials}

Lacprodan 80 (whey protein concentrate) was obtained from Arla Foods Ingredients $\mathrm{P} / \mathrm{S}$ (Brabrand, Denmark). Calcium chloride and pectin extracted from citrus peel (galacturonic acid $\geq 74 \%$ ) were purchased from Sigma Aldrich (St. Louis, MO). Fresh cream containing $38 \%$ fat was purchased in a local supermarket (Arla Foods Ingredients P/S). A commercial chymosin preparation, ChyMaxM1000, was obtained from Chr. Hansen (Hørsholm, Denmark).

\section{Reagents}

Calcium chloride was solubilized in deionized water at a concentration of $54 \mathrm{mM}$, and $9 \%$ (wt/vol) whey solution was prepared by slowly adding the protein to deionized water at $40^{\circ} \mathrm{C}$. Magnetic stirring was applied for $30 \mathrm{~min}$. Pectin was solubilized using the same procedure at a concentration of $1 \%$ (wt/vol). Chymosin was used undiluted in all experiments, corresponding to 8 international milk clotting units (IMCU).

\section{Samples}

Skim milk samples were obtained from Arla Foods Ingredients $\mathrm{P} / \mathrm{S}$. The samples were result of a process
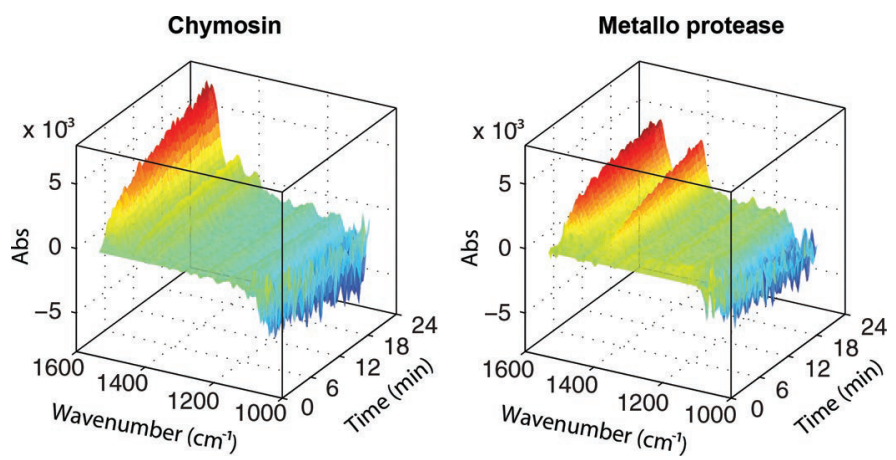

Figure 1. Fourier transform infrared spectra can be measured during the time-course of an enzymatic reaction resulting in a spectral evolution profile (utilizing difference spectra). The spectral evolution profiles for 2 proteases (commercial chymosin and metallo protease from Pectobacterium carotovorum) appear different. Such specific spectral landscapes can be used to distinguish between enzyme activities (including side activities). Color version available online. 
in which relative casein content was increased using a combination of microfiltration and ultrafiltration. The milk was not heated above $42^{\circ} \mathrm{C}$ to prevent denaturation of whey proteins, which is known to cause problems during cheese curd coagulation (Kethireddipalli et al., 2010). Five samples were obtained containing $3.1,3.4,3.7,4.2$, and $5.7 \%$ casein and $3.5,3.9,4.3,4.8$, and $6.5 \%$ total protein, respectively. Milk proteins were quantified using reverse phase chromatography; whey proteins were quantified and subtracted from the total protein amount to yield casein reference data.

Samples were cooled and shipped to the Technical University of Denmark immediately after the protein enrichment experiment. At the university, the samples were stabilized by adding $0.1 \%$ (wt/vol) $\mathrm{NaN}_{3}$ to inhibit bacterial growth and related coagulation. All samples were stored in the refrigerator thereafter, for an experimental period of 2 mo.

\section{Experimental Design}

For the FTIR measurements, an experimental design was set up in which 18 samples were to be measured in 3 replicates, resulting in 54 evolution profiles. The experiment was subdivided into 3: experiment A $(\mathbf{E x p A})$, experiment B (ExpB), and experiment C (ExpC). The processed skim milk samples were diluted as shown in Table 1, yielding variance across 3 different parameters: casein, whey protein, and cream (fat content). Samples for ExpA were diluted with only pectin and water, and samples for $\operatorname{ExpB}$ and $\operatorname{ExpC}$ contained whey additions and whey + cream additions, respectively. Each milk sample was diluted to a total

Table 1. Experimental design: 19 samples (x) were prepared using the dilution scheme below to span variance across casein, whey, fat, and calcium content; experiment (Exp)A, ExpB, and ExpC were used to establish casein calibration models, ExpD was used as a mini-test set to illustrate calcium robustness of the model $^{1}$

\begin{tabular}{|c|c|c|c|c|c|c|c|}
\hline \multirow[b]{2}{*}{ Sample } & \multirow{2}{*}{$\frac{\text { ExpA }}{\text { A0 }}$} & \multicolumn{2}{|c|}{ ExpB } & \multicolumn{3}{|c|}{ ExpC } & \multirow{2}{*}{$\frac{\operatorname{ExpD}}{\mathrm{A} 0 \mathrm{R}}$} \\
\hline & & $\mathrm{A} 1$ & $\mathrm{~A} 2$ & $\mathrm{~A} 0 \mathrm{C}$ & $\mathrm{A} 1 \mathrm{C}$ & $\mathrm{A} 2 \mathrm{C}$ & \\
\hline 1 & $\mathrm{x}$ & $\mathrm{x}$ & $\mathrm{x}$ & $\mathrm{x}$ & & $\mathrm{x}$ & \\
\hline 2 & $\mathrm{x}$ & & $\mathrm{x}$ & $\mathrm{x}$ & & & \\
\hline 3 & $\mathrm{x}$ & $\mathrm{x}$ & & & $\mathrm{x}$ & & $\mathrm{x}$ \\
\hline 4 & $x$ & $\mathrm{x}$ & $\mathrm{x}$ & & & $\mathrm{x}$ & \\
\hline 5 & $\mathrm{x}$ & $\mathrm{x}$ & & & $\mathrm{x}$ & & \\
\hline
\end{tabular}

${ }^{1}$ All milk dilutions consisted of $20 \mathrm{~mL}$ of sampled skim milk and $5 \mathrm{~mL}$ of pectin solution. To reach a total volume of $35 \mathrm{~mL}$, the following constituents were added: $\mathrm{A} 0=10 \mathrm{~mL}$ of $\mathrm{H}_{2} \mathrm{O}$; $\mathrm{A} 1=4 \mathrm{~mL}$ of whey solution $+6 \mathrm{~mL}$ of $\mathrm{H}_{2} \mathrm{O} ; \mathrm{A} 2=8 \mathrm{~mL}$ of whey solution $+2 \mathrm{~mL}$ of $\mathrm{H}_{2} \mathrm{O}$; $\mathrm{A} 0 \mathrm{C}=2 \mathrm{~mL}$ of cream $+8 \mathrm{~mL}$ of $\mathrm{H}_{2} \mathrm{O} ; \mathrm{A} 1 \mathrm{C}=4 \mathrm{~mL}$ of whey solution $+2 \mathrm{~mL}$ of cream $+4 \mathrm{~mL}$ of $\mathrm{H}_{2} \mathrm{O} ; \mathrm{A} 2 \mathrm{C}=8 \mathrm{~mL}$ of whey solution +2 $\mathrm{mL}$ of cream; $\mathrm{A} 0 \mathrm{R}=1 \mathrm{~mL}$ of $\mathrm{CaCl}_{2}$ solution $+9 \mathrm{~mL}$ of $\mathrm{H}_{2} \mathrm{O}$. volume of $35 \mathrm{~mL}$. The reference result for the $\operatorname{ExpC}$ samples had to be adjusted because the cream also contained a significant amount of casein. In addition to the described experimental design, 1 sample containing added $\mathrm{CaCl}_{2}$ was measured (2 replicates) in experiment D (ExpD). This sample was added to the experiments to illustrate calibration robustness against calcium. It was not part of the calibration modeling, but was used as a mini-test set instead.

Pectin was added to all samples, because initial studies had shown that it stabilized casein coagulation in skim milk (data not shown). These initial findings were in accordance with phenomena described in the literature (Liu et al., 2008; Lobato-Calleros et al., 2008), by which pectin can be understood as a hydrocolloid that helps to stabilize cheese curd texture during coagulation.

\section{Experimental Procedure}

All spectra were acquired using a MilkoScan FT2 (Foss Analytical, Hillerød, Denmark). The instrument consisted of an FTIR that scanned the mid-infrared part of the electromagnetic spectrum. Acquisition was carried out using the method described in Andersen, 2002, with an optical resolution of $14 \mathrm{~cm}^{-1}$. The instrument was equipped with an automatic flow-through system and worked semi-automatically. The optical system was hermetically sealed, and it was pressure-, temperature-, and humidity-controlled. The path length of the cuvette was $50 \mu \mathrm{m}$.

Prior to measurements, samples were preheated to the cuvette temperature of $42^{\circ} \mathrm{C}$ to avoid spectral artifacts along the temporal domain (i.e., in the spectral region around $1,150 \mathrm{~cm}^{-1}$ ) due to relative heat shock as they were pumped into the heat exchanger. Samples were measured in random order.

To initialize the acquisition of an evolution profile, every sample mixture was gently shaken, and $10 \mathrm{~mL}$ were pumped into the flow-through system to prepare the instrument for the chemical matrix of the sample. Thereafter, the remaining sample amount was mixed with $8 \mu \mathrm{L}$ of the enzyme ( $8 \mathrm{IMCU})$. The sample was gently shaken again, and acquisition of the evolution profile was initiated by pumping $10 \mathrm{~mL}$ of the enzymatically spiked sample into the instrument. Spectra were acquired consecutively, immediately after intake, with a rate of 3.61 spectra/min. Fifty spectra were acquired (13.8 min evolution time) while the sample mixture remained inside the cuvette. All spectra were measured against a water reference spectrum. After each evolution profile acquisition, the flow-through system was cleaned using an automatic cleaning program. 


\section{Data Handling and Analysis}

All data were exported using Foss Integrator (version 1.5.3; Foss Analytical, Hillerød, Denmark) and handled using Matlab (The MathWorks Inc., Natick, MA). Chemometric multiway analysis was performed using PLS Toolbox (version 8.0.2; Eigenvector Research Inc., Manson, WA; Wise et al., 2006).

Data Structure. Information from the fingerprint region of the infrared spectrum was used for visualization and chemometric analysis; this corresponded to wavenumbers from 1,000 to $1,600 \mathrm{~cm}^{-1}$. Each evolution profile was represented by a data matrix $(M \times N)$, indicating $M$ spectral wavenumbers and $N$ number of spectra (evolution time) along its dimensions. Because all evolution profiles were measured using the same consecutive time steps, the individual matrices could be stacked to form a 3-way data structure $(L \times M \times N)$, indicating $L$ samples, $M$ spectral wavenumbers, and $N$ number of spectra. The resulting tensor [54 samples $\times 143$ wavenumbers $\times 50$ spectra (time steps)] was the starting point for multiway analysis/calibration (tensor decomposition). See Baum et al. (2013b) for additional information on how the multiway data structure was formed.

Data Visualization. Difference spectra were calculated to subtract the constant spectral background. By doing so, the first spectrum was subtracted from the entire series, resulting in an evolution profile. Because it was subtracted from itself, the first spectrum would result in a zero line; subsequent spectra showed changes in relation to the spectrum at time $=0$.

Data Preprocessing. All evolution profiles were prepared for analysis by calculating difference spectra in the same way as for visualization. We then applied multiway centering across the sample and the spectral mode to remove unwanted offsets that might lead to complications when performing multiway analysis. To center the tensor (e.g., across its sample mode), it was unfolded to an $L \times M N$ matrix. Thereafter, we proceeded as in usual 2-way analysis as described in Equation [1] (Bro, 1997; Bro and Smilde, 2003):

$$
x_{l m n}^{c e n t}=x_{l m n}-\frac{\sum_{l=1}^{L} x_{l m n}}{L},
$$

where $l=1, \ldots, L ; m=1, \ldots, M ; n=1, \ldots, N$. Evolution profiles before and after spectral mode centering are shown in Figure 2. Spectral mode centering removed constant spectral offsets that arose due to scattering effects in the sample.

Multiway Methods and Figures of Merit. The PARAFAC and NPLS methods were applied to per- form multiway analysis. See Baum et al. (2013a), Bro (1997), Bro (2006), Escandar et al. (2007), and Faber (2015) for further information on chemometric methods. Models were established using contiguous block cross-validation (data splits $=18$ samples; block size $=3$ replicates) by excluding all 3 replicates of each sample simultaneously to avoid unwanted bias (Westad and Marini, 2015). The root mean square error of calibration (RMSEC) was calculated as described in Equation [2]:

$$
R M S E C=\sqrt{\frac{\sum_{l=1}^{L}\left(y_{l}-\widehat{y}_{l}\right)^{2}}{L}},
$$

where $y_{l}=$ predicted casein concentration of the $l$ th sample; and $\hat{y}_{l}=$ known casein concentration of the lth sample. The corresponding root mean square error of cross-validation (RMSECV) was calculated using the same formalism, and casein concentrations $y_{l}$ were predicted from the excluded samples using the respective model in each validation step. The precision of each model was calculated by simply averaging the standard deviation across all sample predictions.

\section{RESULTS AND DISCUSSION}

\section{Effect of Casein on Spectral Evolution}

When analyzing the data, our first step was to visualize the spectra to achieve an understanding of apparent changes in relation to the underlying enzymatic chemistry (perturbation). Figure 3 shows 3 acquired evolution profiles from ExpA. All samples varied in casein content, and the intensity of the spectral evolution increased with casein concentration, because of different reaction


Figure 2. We used spectral mode centering to remove niveau shifts over time. Such offsets can arise due to scattering effects. Left: spectral evolution without spectral mode centering (raw); right: same spectral evolution after spectral mode centering (preprocessed). Color version available online. 

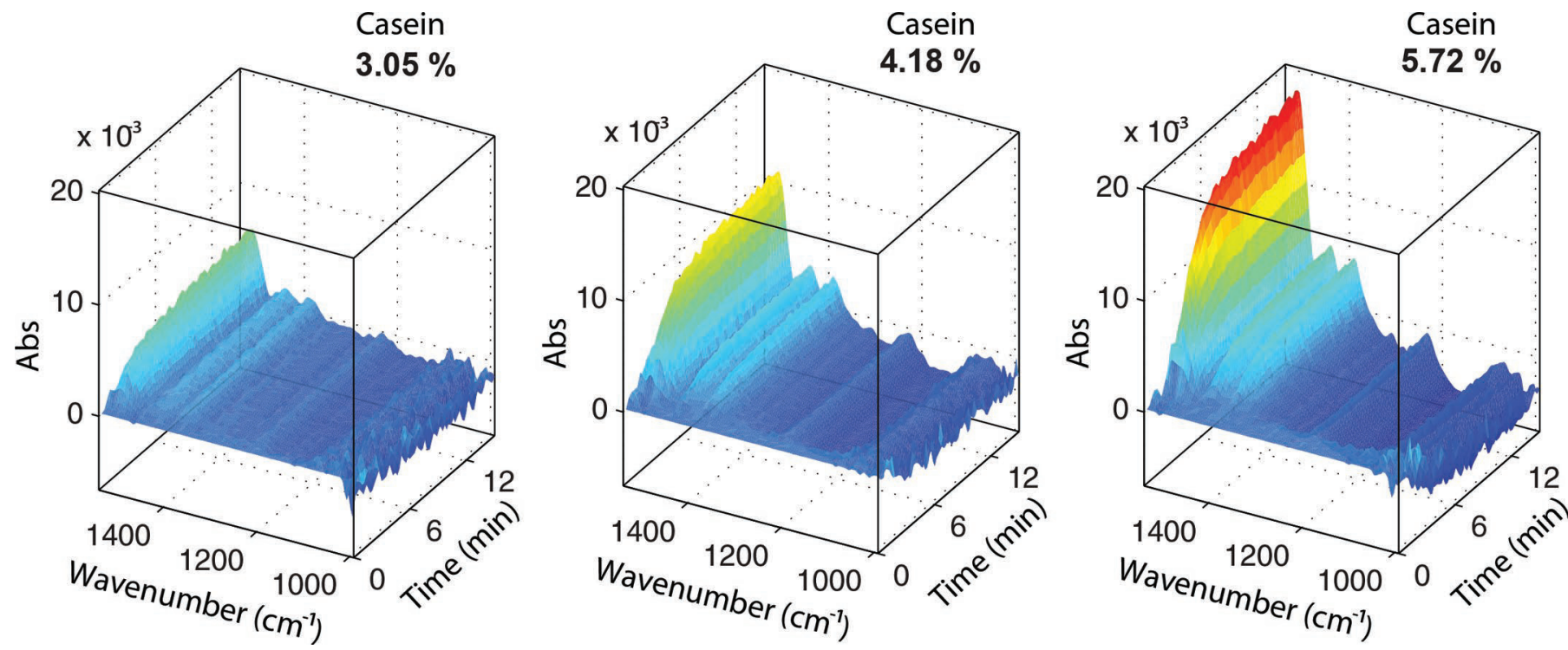

Figure 3. Equal amounts of chymosin were used to induce spectral evolution in samples containing different amounts of casein. It can clearly be observed that the intensity of the spectral evolution depends on the casein content in the milk solution. Color version available online.

rates for enzymatic hydrolysis along the temporal domain. Certain spectral bands increased in intensity over time [e.g., the protein amid II band around $1,500 \mathrm{~cm}^{-1}$ (Kong and Yu, 2007)] and others decreased. Besides that the reaction rate differed between samples, the spectral change converged at different levels related to the underlying casein concentrations.

We also observed that casein concentration had a direct influence not only on the intensity of spectral evolution, but also on the effectiveness of cheese curd coagulation. Therefore, it could be concluded that we monitored real cheese-making (i.e., casein coagulation).

\section{Robustness}

Figure 4 illustrates 4 evolution profiles for samples that contained the same casein concentration. The sample in Figure 4A contained $740 \mathrm{mg}$ of casein and 50 $\mathrm{mg}$ of pectin, and the reaction was induced by $8 \mathrm{IMCU}$ of chymosin. In Figures 4B, C, and D, samples were spiked with whey protein, $\mathrm{CaCl}_{2}$, and whey + cream, respectively.

Spectral evolution was not affected by the addition of whey protein or $\mathrm{CaCl}_{2}$ : the evolution profiles were visually similar in Figures $4 \mathrm{~A}, \mathrm{~B}$, and $\mathrm{C}$, indicating that the measurements were robust against whey protein and calcium. However, when cream was added to the solution (Figure 4D), a second type of kinetic change appeared in the spectra, at $1,100 \mathrm{~cm}^{-1}$, increasing the mathematical rank of the data. This additional spectral evolution superimposed the casein coagulation-related evolution. However, such superimpositions of enzymati-


\section{C $740 \mathrm{mg}$ casein $+50 \mathrm{mg}$ pectin $+6 \mathrm{mg} \mathrm{CaCl}$}


\section{D $740 \mathrm{mg}$ casein $+50 \mathrm{mg}$ pectin $+360 \mathrm{mg}$ whey + $1 \mathrm{ml}$ cream (38\% fat)}

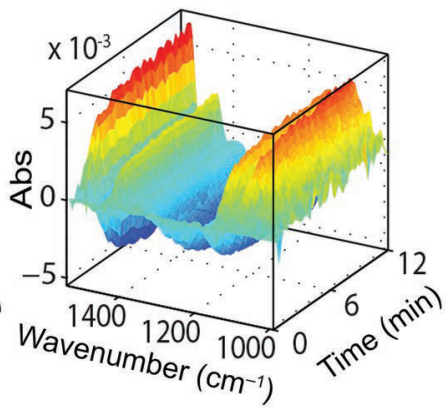

Figure 4. Chymosin was added to milk solutions containing equal amounts of casein, but different amounts of whey protein, calcium, and fat. The spectral evolution is robust against varying whey and calcium levels as it can be seen from A, B, and C. However, the addition of fat (cream) induced a second type of spectral change which superimposed the first one (D). Pectin was added to all solutions as a cheese curd stabilizer. Color version available online. 
cally induced spectral changes could be resolved using multivariate curve resolution (Amigo et al., 2006) or chemometric tensor decomposition methods, such as PARAFAC, Tucker3 or NPLS (Pettersson and Karlberg, 1997; Baum et al., 2013a).

When measuring the same sample (740 mg of casein) without added pectin, the spectral evolution decreased significantly (as did the observable cheese curd coagulation). On the other hand, the addition of whey led to enhanced spectral evolution when pectin was not present (data not shown). Pectin was therefore essential for obtaining the robust results shown in Figure 4. As reported previously, the presence of pectin during coagulation changes the structural properties of lowfat cheeses by forming gels (Liu et al., 2008; LobatoCalleros et al., 2008). In this way, the pectin is seen to interact with the protein matrix of the coagulate (Liu et al., 2008; Lobato-Calleros et al., 2008).

\section{Casein Calibration}

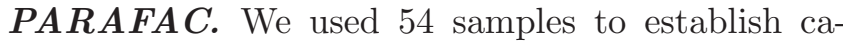
sein-specific calibration models using enzyme-induced perturbation. Besides the previously mentioned spectral evolution related to casein (Figure 3), we detected no other spectral changes for samples in ExpA and ExpB, indicating a single component for analysis using PARAFAC. However, in ExpC, which included samples with added cream, we found a superimposition of 2 spectral changes (Figure 4D). In this case, we expected 2 components for analysis using PARAFAC.

Model details for a 2-component PARAFAC model that contained all 54 samples from ExpA, ExpB, and ExpC are shown in Figures 5A-C. The model was highly unique because a core consistency of $100.00 \%$ indicated a super diagonal core tensor $G$. See Bro and Kiers (2003) for additional information on core consistency diagnostics and how to choose the right number of components. All 54 samples indicated low residuals (Figure 5A), indicating that the entire kinetic-induced variance in the spectra could be captured using the model (91.2\% variance). The scores for the samples (Figure 5B) indicated that casein-related evolution was described mainly by component 2 ; component 1 was necessary to describe variance in the cream-related samples (ExpC). Therefore, we established a crossvalidated calibration model using the component 2 scores (Figure 5C). Although we observed satisfying calibration performance for ExpA and ExpB samples, we found low robustness for the addition of cream. We observed an intercept and less significant slope offsets for all cream samples, leading to unsatisfying calibration performance in terms of RMSEC (0.29\%), RMSECV (0.34\%), and $\mathrm{R}^{2}(0.87)$. Alternatively, the 2


Figure 5. Parallel factor analysis (PARAFAC) model details: (A) sample mode residuals (sum of squares, SSQ); (B) score plot; (C) cross-validated $(\mathrm{CVL})$ calibration. RMSEC $=$ root mean square error of calibration; RMSECV = root mean square error of cross-validation; $\operatorname{Exp}=$ experiment ; Comp $=$ component. Color version available online.

components could be combined using multiple linear regression, yielding a model that indicated the following figures of merit: RMSEC (0.10\%), RMSECV (0.12\%), and $\mathrm{R}^{2}(0.98)$, with a corresponding precision of $0.07 \%$. This approach is comparable to applying multiple linear regression to principal component analysis scores in chemometric 2-way analysis, which is referred to as principal component regression.

Our good results when we combined the 2 components using multiple linear regression indicated that there was interaction between the 2 components and explained why PARAFAC could not resolve the 2 underlying spectral profiles. For this reason, we created models using NPLS.

NPLS. We used all 54 samples to establish an NPLS calibration model using 2 latent variables (components), as indicated from the evolution profiles in Figure 4. We confirmed the assumption that 2 latent variables were necessary to describe the variance in the data by plotting the RMSECV error over the component number. An increase in calibration error (RMSECV) when more than 2 latent variables were used indicated overfitting (Figure 6A).

The spectral and kinetic mode loadings were comparable to the PARAFAC results (Figure $6 \mathrm{C}+\mathrm{D}$ ), leading to similar patterns in the score plot (Figure 6B). In 
A
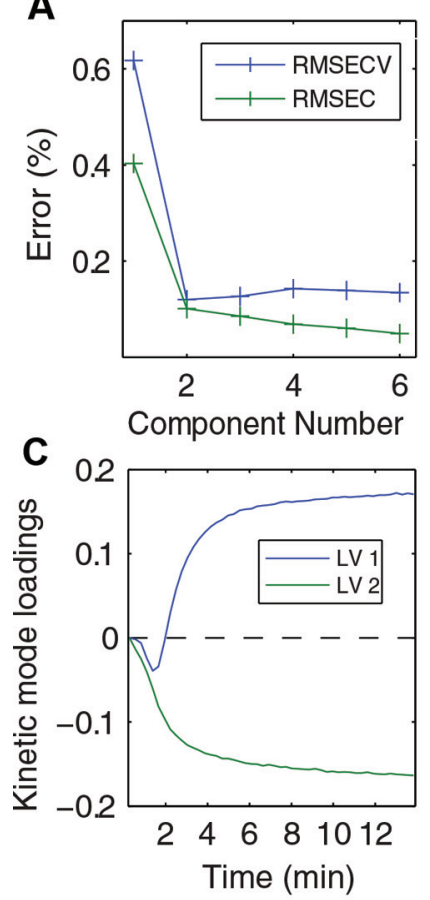

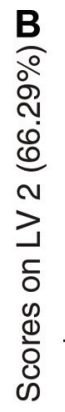

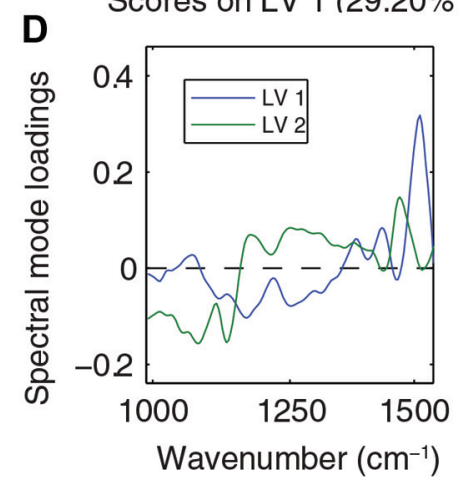

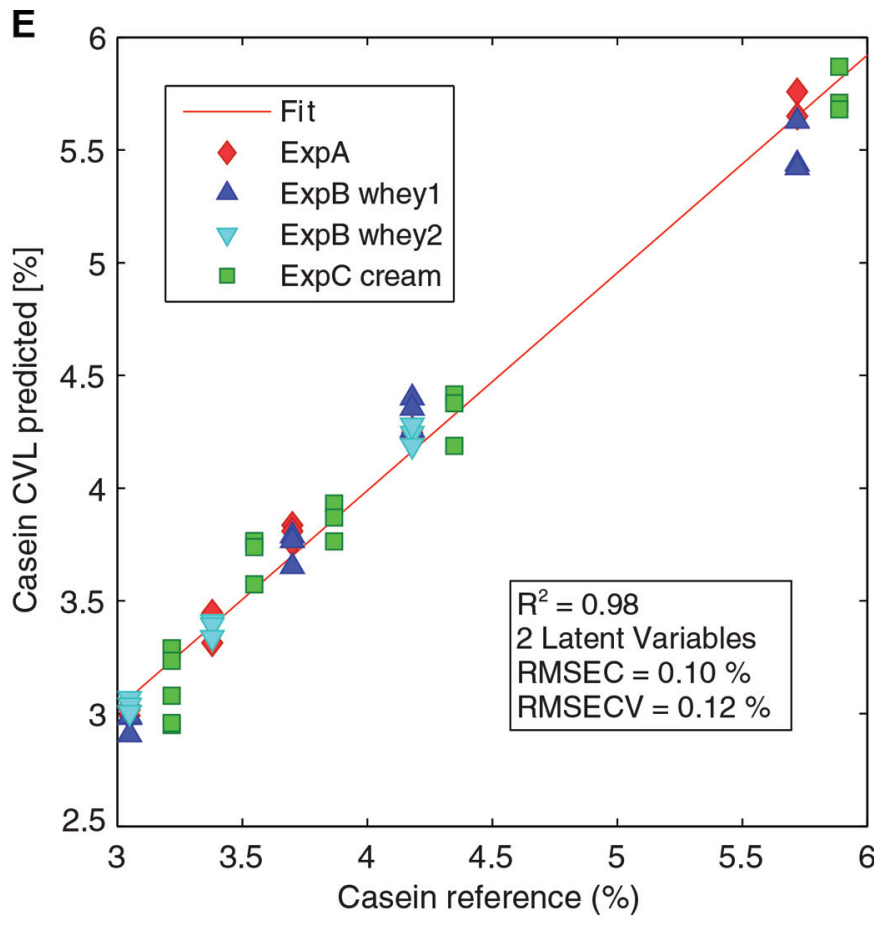

\section{F Component \#1}

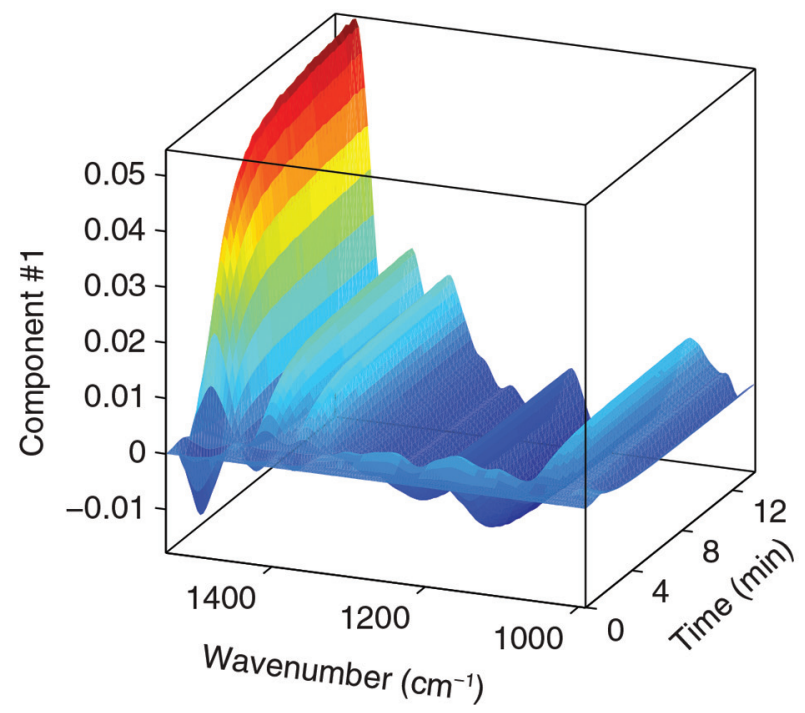

G Component \#2

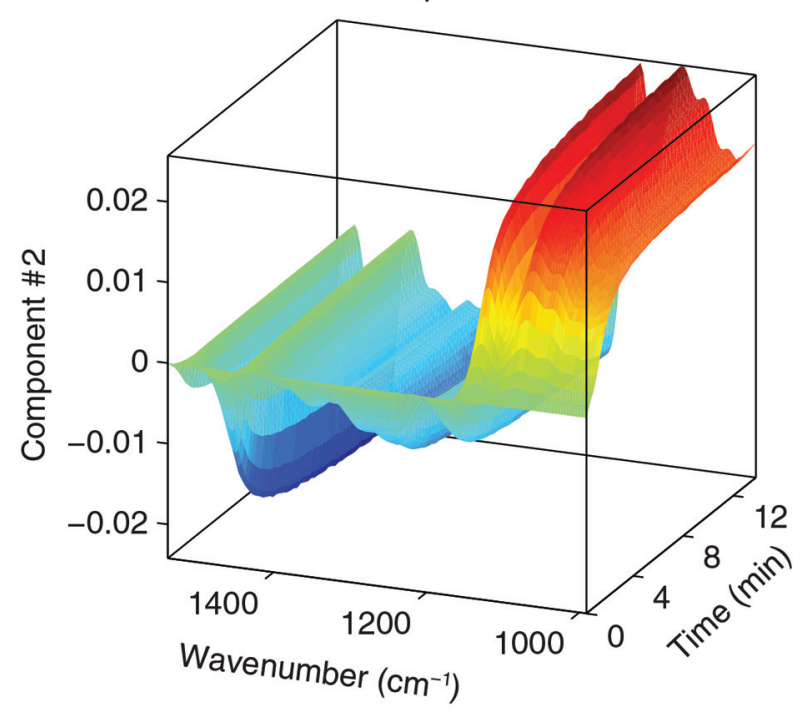

Figure 6. Multiway partial least squares (NPLS) model details: (A) root mean square error of calibration (RMSEC) and root mean square error of cross-validation (RMSECV) in dependency of number of components; (B) score plot; (C) kinetic mode loadings; (D) spectral mode loadings; (E) cross-validated (CVL) calibration; (F) loading surface component 1; (G) loading surface component 2. Exp = experiment; LV = latent variable. Color version available online.

contrast to unsupervised PARAFAC analysis, NPLS is a supervised tensor decomposition method and therefore combined scores from both latent variables using linear regression. We calculated the loading surfaces for both components as the outer vector products of kinetic and spectral loadings; results are displayed in Figure $6 \mathrm{~F}+\mathrm{G}$. The cream-induced spectral change could be resolved in component 2 . Figure $6 \mathrm{E}$ presents the resulting NPLS calibration, indicating the following figures of merit: RMSEC (0.10\%), RMSECV (0.12\%), and $R^{2}(0.98)$. Repeatability (precision) was $0.07 \%$.

Because only a combination of both components led to satisfactory calibration performance, we confirmed interaction of the 2 loadings. One possible reason for 
this may have been interaction between the added fat of the cream and the casein during coagulation. Fat is typically incorporated in the cheese curd and could explain the interaction between the 2 factors.

To ensure the robustness of the established model against calcium, we spiked 1 sample with $\mathrm{CaCl}_{2}$ (ExpD). We measured 2 replicates and predicted casein content using the NPLS calibration model. The respective evolution profile is shown in Figure $4 \mathrm{C}$. The mean prediction error was $0.12 \%$, in accordance with the reported accuracy (RMSECV) of the model and indicating robustness against $\mathrm{CaCl}_{2}$.

\section{CONCLUSIONS}

We have introduced a rapid and inexpensive tool for quantifying casein in subcritical heated skim milk using FTIR and enzymatic perturbation. Measurements were carried out online and monitored the cheese-makingrelevant casein via specific hydrolysis induced by chymosin. The resulting kinetic evolution profiles could be analyzed using tensor decomposition methods such as PARAFAC and NPLS, yielding calibration models with robustness against calcium, whey, and cream. The accuracy and precision of the models were 0.12 and $0.07 \%$, respectively. One analysis could be performed in about 13 min and could be optimized further. In contrast to conventional multivariate calibrations, the suggested methodology relied on direct measurement of cheesemaking-relevant casein instead of indirect calibration effects, which might bias the result due to underlying covariance effects. Because the established calibrations focused solely on kinetic change, the chemical background was irrelevant and could be subtracted from the data. Hence, the established calibrations yielded the second-order advantage known from chemometric multiway analysis.

\section{ACKNOWLEDGMENTS}

This work was financed by Foss Analytical (Hillerød, Denmark). We acknowledge Arla Foods (Brabrand, Denmark) for providing the skim milk samples and the HPLC reference analysis.

\section{REFERENCES}

Amigo, J. M., A. de Juan, J. Coello, and S. Maspoch. 2006. A mixed hard- and soft-modelling approach for the quantitative determination of oxipurines and uric acid in human urine. Anal. Chim. Acta 567:236-244. http://dx.doi.org/10.1016/j.aca.2006.03.041.

Andersen, S. K., P. W. Hansen, and H. V. Andersen. 2002. Vibrational spectroscopy in the analysis of dairy products and wine. In Handbook of Vibrational Spectroscopy. J. M. Chalmer and P. R. Griffiths ed. John Wiley \& Sons, Chichester, UK.
Baum, A., P. W. Hansen, A. S. Meyer, and J. D. Mikkelsen. 2013a Simultaneous measurement of two enzyme activities using infrared spectroscopy: A comparative evaluation of PARAFAC, Tucker and N-PLS modeling. Anal. Chim. Acta 790:14-23. http://dx.doi. org/10.1016/j.aca.2013.06.039.

Baum, A., A. S. Meyer, J. L. Garcia, M. Egebo, P. W. Hansen, and J. D. Mikkelsen. 2013b. Enzyme activity measurement via spectral evolution profiling and PARAFAC. Anal. Chim. Acta 778:1-8.

Baum, A., L. Nørgaard, P. W. Hansen, H. V. Juhl, and J. D. Mikkelsen. 2016. Determination of a constituent related property of a multiconstituent sample. Int. Pat. Appl. No. PCT/EP2014/067549. Foss Analytical A/S, assignee.

Berhe, D. T., C. E. Eskildsen, R. Lametsch, M. S. Hviid, F. van den Berg, and S. B. Engelsen. 2016. Prediction of total fatty acid parameters and individual fatty acids in pork backfat using Raman spectroscopy and chemometrics: Understanding the cage of covariance between highly correlated fat parameters. Meat Sci. 111:18 26. http://dx.doi.org/10.1016/j.meatsci.2015.08.009.

Bro, R. 1997. PARAFAC. Tutorial and applications. Pages 149-171 in Chemometrics and Intelligent Laboratory Systems, vol. 38.

Bro, R. 2006. Review on multiway analysis in chemistry - 2000-2005. Crit. Rev. Anal. Chem. 36:279-293. http://dx.doi. org $/ 10.1080 / 10408340600969965$.

Bro, R., and H. A. L. Kiers. 2003. A new efficient method for determining the number of components in PARAFAC models. J. Chemometr. 17:274-286. http://dx.doi.org/10.1002/cem.801.

Bro, R., and A. K. Smilde. 2003. Centering and scaling in component analysis. J. Chemometr. 17:16-33. http://dx.doi.org/10.1002/ cem.773.

Escandar, G.M., A.C. Olivieri, N. (Klaas) M. Faber, H.C. Goicoechea, A. Muñoz de la Peña, and R.J. Poppi. 2007. Second- and third-order multivariate calibration: Data, algorithms and applications. TrAC. Trends Analyt. Chem. 26:752-765. http://dx.doi. org/10.1016/j.trac.2007.04.006.

Eskildsen, C. E., M. A. Rasmussen, S. B. Engelsen, L. B. Larsen, N. A. Poulsen, and T. Skov. 2014. Quantification of individual fatty acids in bovine milk by infrared spectroscopy and chemometrics: Understanding predictions of highly collinear reference variables. J. Dairy Sci. 97:7940-7951. http://dx.doi.org/10.3168/jds.2014-8337.

Etzion, Y., R. Linker, U. Cogan, and I. Shmulevich. 2004. Determination of protein concentration in raw milk by mid-infrared Fourier transform infrared/attenuated total reflectance spectroscopy. J. Dairy Sci. 87:2779-2788. http://dx.doi.org/10.3168/jds.S00220302(04)73405-0.

Faber, N. M. 2015. Fundamentals and Analytical Applications of Multiway Calibration. Elsevier, Amsterdam, the Netherlands.

Feng, T., C. Nyffenegger, P. Højrup, S. Vidal-Melgosa, K. P. Yan, J. U. Fangel, A. S. Meyer, F. Kirpekar, W. G. Willats, and J. D. Mikkelsen. 2014. Characterization of an extensin-modifying metalloprotease: N-terminal processing and substrate cleavage pattern of Pectobacterium carotovorum Prt1. Appl. Microbiol. Biotechnol. 98:10077-10089.

Hewavitharana, A. K., and B. van Brakel. 1997. Fourier transform infrared spectrometric method for the rapid determination of casein in raw milk. Analyst (Lond.) 122:701-704. http://dx.doi. org/10.1039/a700953d.

Kethireddipalli, P., A. R. Hill, and D. G. Dalgleish. 2010. Protein interactions in heat-treated milk and effect on rennet coagulation. Int. Dairy J. 20:838-843. http://dx.doi.org/10.1016/j. idairyj.2010.06.003.

Kong, J., and S. Yu. 2007. Fourier transform infrared spectroscopic analysis of protein secondary structures. Acta Biochim. Biophys. Sin. (Shanghai) 39:549-559.

Langholm Jensen, J., A. Mølgaard, J.-C. Navarro Poulsen, M. K. Harboe, J. B. Simonsen, A. M. Lorentzen, K. Hjernø, J. M. van den Brink, K. B. Qvist, and S. Larsen. 2013. Camel and bovine chymosin: The relationship between their structures and cheese-making properties. Acta Crystallogr. D Biol. Crystallogr. 69:901-913. http://dx.doi.org/10.1107/S0907444913003260.

Liu, H., X. M. Xu, and S. D. Guo. 2008. Comparison of full-fat and low-fat cheese analogues with or without pectin gel through micro- 
structure, texture, rheology, thermal and sensory analysis. Int. J. Food Sci. Technol. 43:1581-1592.

Lobato-Calleros, C., A. Sosa-Pérez, J. Rodríguez-Tafoya, O. SandovalCastilla, C. Pérez-Alonso, and E. J. Vernon-Carter. 2008. Structural and textural characteristics of reduced-fat cheese-like products made from W1/O/W2 emulsions and skim milk. LWT Food Sci. Technol. (Campinas) 41:1847-1856. http://dx.doi.org/10.1016/j. lwt.2008.01.006.

Lucey, J. A., and P. F. Fox. 1993. Importance of calcium and phosphate in cheese manufacture: A review. J. Dairy Sci. 76:1714-1724. http://dx.doi.org/10.3168/jds.S0022-0302(93)77504-9.

Pettersson, A. K., and B. Karlberg. 1997. Simultaneous determination of orthophosphate and arsenate based on multi-way spectroscopickinetic data evaluation. Anal. Chim. Acta 354:241-248. http:// dx.doi.org/10.1016/S0003-2670(97)00419-4.

Susi, H., and D. M. Byler. 1986. Resolution-enhanced Fourier transform infrared spectroscopy of enzymes. Methods Enzymol. 130:290-311.

Venyaminov, S. Yu., and N. N. Kalnin. 1990. Quantitative IR spectrophotometry of peptide compounds in water $\left(\mathrm{H}_{2} \mathrm{O}\right)$ solutions. II. Amide absorption bands of polypeptides and fibrous proteins in alpha-, beta-, and random coil conformations. Biopolymers 30:1259-1271.

Wedholm, A., L. B. Larsen, H. Lindmark-Månsson, A. H. Karlsson, and A. Andrén. 2006. Effect of protein composition on the cheese-making properties of milk from individual dairy cows. J. Dairy Sci. 89:3296-3305. http://dx.doi.org/10.3168/jds.S00220302(06)72366-9.

Westad, F., and F. Marini. 2015. Validation of chemometric models-A tutorial. Anal. Chim. Acta 893:14-24. http://dx.doi. org/10.1016/j.aca.2015.06.056.

Wise, B. M., N. B. Gallagher, R. Bro, J. M. Shaver, W. Windig, and R. S. Koch. 2006. PLS_Toolbox Version 4.0 for use with Matlab. Eigenvector Technologies, Manson, WA.

Xie, L.-X., H.-L. Wu, Y. Fang, C. Kang, S.-X. Xiang, L. Zhu, X.-L. Yin, H.-W. Gu, Z. Liu, and R.-Q. Yu. 2015. Simultaneous determination of tyrosine and levodopa in human plasma using enzyme-induced excitation-emission-kinetic third-order calibration method. Chemom. Intell. Lab. Syst. 148:9-19. http://dx.doi.org/10.1016/j. chemolab.2015.08.023. 